\title{
Relationship between the Morphology and Mechanical Properties of Hydroxypropyl Cellulose Films and the Characteristic Behavior of the Chains in Solution
}

\author{
Naoko Yanagida, Satomi Takematsu, and Masaru Matsuo* \\ Department of Textile and Apparel Science, Faculty of Human Life and Environment, \\ Nara Women's University, Nara 630 Japan
}

(Received June 30, 1992)

\begin{abstract}
The morphology and drawability of hydroxypropyl cellulose (HPC) films cast from water, $N, N$-dimethyl formamide, and acetic acid solutions were investigated in terms of the molecular weight and characteristics of the HPC chains in solution. Liquid crystals showing cholesteric color were observed in water and acetic acid solutions with appropriate concentrations, where the weight average degree of polymerization $\left(\overline{D P}_{w}\right)$, is less than 370 . Interestingly, the color was maintained within films cast from acetic acid. In constant, the color could not be observed for HPC chains with $\overrightarrow{D P}>1750$. These phenomena were investigated by elastic and inelastic light scattering, polarized small-angle light scattering and optical microscopy. Interestingly, the HPC chains of $\overrightarrow{D P}<370$ were found to be stiffest in acetic acid. The characteristics of the HPC chains in solvent also significantly effected on the drawability of resultant cast films. That is, the drawability of the film was sensitive to the molecular weight of the HPC and the type of solvent. The light scattering measurements showed that the drawability becomes more pronounced as the HPC chain expansion in solvent increases.
\end{abstract}

KEY WORDS Hydroxypropyl Cellulose/Solution / Liquid Crystals / Degree of Polymerization / Elastic and Inelastic Light Scattering /

Hydroxypropyl cellulose (HPC) is a watersoluble derivative of cellulose with poly(1,2alkyleneoxide) side chains. The morphology and deformation mechanism of HPC films cast from water were first investigated in detail by Samuls $^{1}$ at all levels from molecular to superstructural by X-ray diffraction, smallangle light scattering, birefringence and infrared measurements. More recently, the molecular weight dependence of morphology and mechanical properties of HPC films cast from water was discussed by Matsuo and Yanagida ${ }^{2}$ using samples with three molecular weights. The results indicated that the drawability become more pronounced with increasing molecular weight. Furthermore, light scattering patterns from concentrated solutions

\footnotetext{
* To whom correspondence should be addressed.
}

under $H_{\mathrm{v}}$ polarization condition was found to be sensitive to molecular weight. The light scattering pattern of the specimen with the weight-average molecular weight, $19.2 \times 10^{4}$, showed +-type lobes at small angles and $x$-type at large angles, reflecting interparticle interference effects of rods during the evaporation of solvent, but the $\times$-type lobes disappeared when the solvent was completely evaporated. Such behavior, however, was not observed for a specimen with $\bar{M}_{w}=119.5 \times 10^{4}$ and the pattern showed only +-type lobes, indicating the scattering from lobes, the optical axes being oriented at $40-50^{\circ}$ with respect to the rod axis. ${ }^{3}$

Here it should be noted that HPC molecules can be dissolved in several kinds of solvents at 
room temperature. Thus, HPC samples provide suitable materials to study the relationship between characteristics of macromolecules in solution and the resultant film after evaporation of solvent. This is one of the most important subjects for polymer science but remains an uniresolved problem. For example, polyethylene is one of the most common polymers and the improvement of the mechanical properties has been studied from the viewpoint that the maximum attainable draw ratio depends on the number of entanglements per molecule in solution. ${ }^{4}$ The characteristics of polyethylene molecules in solution, however, have never been studied in detail, for example, by elastic and inelastic light scattering, since polyethylene molecules can't be dissolved in $p$-xylene and decalin at temperatures less than $80^{\circ} \mathrm{C}$. Accordingly, this paper deals with the morphology and mechanical properties of bulk materials in relation to characteristics of HPC chains in solution. The best way to pursue this problem is to use the sample whose molecular weight distribution is narrow. A solutionfractionation technique for HPC has been developed by Wirick and Waldman, ${ }^{5}$ utilizing mixtures of anhydrous ethanol and $n$-heptane of varying composition. Unfortunately, it turned out that amount of HPC powder obtained by their method is too small to prepare cast films for the purpose of studying morphology and mechanical properties. Hence, we have been obliged to study the characteristics of HPC chains in solution using HPC powders with a broad molecular weight distribution.

\section{EXPERIMENTAL}

As in the previous work, ${ }^{2}$ three kinds of samples listed in Table I were employed as test specimens. The degree of substitution of the HPC is expressed in terms of the molar substitution (MS). As the weight-avrage molecular weight, $\bar{M}_{w}$, and the number-average molecular weight, $\bar{M}_{n}$, the value estimated by
Table I. Characterization of the HPC specimens

\begin{tabular}{cccc}
\hline Sample code & $M_{\mathrm{s}}$ & $\bar{M}_{w}\left(\times 10^{4}\right)$ & $\bar{M}_{n}\left(\times 10^{4}\right)$ \\
\hline A & 4.25 & 11.7 & 5.2 \\
B & 3.48 & 19.2 & 8.9 \\
C & 3.37 & 119.5 & 26.8 \\
\hline
\end{tabular}

Suto of Yamagata University using gel permeation chromatography were employed here.

In the present experiments, water, ethanol, $N, N$-dimethylformamide (DMF), and acetic acid were used as solvents to study the effect of the HPC chain characteristics in solution on the morphology and mechanical properties of the resultant films. HPC films were prepared by casting in a flat petri dish from solutions of concentration $10 \mathrm{~g} / 100 \mathrm{ml}$ for specimens $\mathrm{A}$ and $B$ and $2.5 \mathrm{~g} / 100 \mathrm{ml}$ for specimen, $C$ with subsequent air drying at room temperature. The resultant films with various thicknesses were vacuum-dried for 2 days to remove residual traces of solvent.

The dry films were cut into strips of length $70 \mathrm{~mm}$ and width $10 \mathrm{~mm}$. The strips were clamped in a manual device in such a way that the length to be drawn was $50 \mathrm{~mm}$. The specimens was placed in a hot oven at 150 $170^{\circ} \mathrm{C}$ under nitrogen and elongated manually to the desired draw ratios after annealing for $15 \mathrm{~min}$. After stretching, the sample retained in the stretcher was annealed at the elongation temperature for $10 \mathrm{~min}$ and cooled slowly to room temperature.

Photon correlation measurements were carried out by using an inelastic light scattering instrument (DLS-700 manufactured by Ohtsuka Electric Co., Ltd.) at $20^{\circ} \mathrm{C}$. The detailed method to estimate the autocorrelation function has been discussed elsewhere. ${ }^{6}$ The elastic light scattering measurements were also carried out with the same instrument (DLS700). On the measurements of elastic light scattering, the differential values of refractive index increment to concentration $C, \mathrm{~d} n / \mathrm{d} c$, are determined to be 0.1021 and 0.1134 for water 
and acetic acid, respectively, using differential reflectometer (RM-102 manufactured by Ohtsuka Electric Co., Ltd.). On the measurements of inelastic light scattering, the solvent viscosities of water, acetic acid and DMF at $20^{\circ} \mathrm{C}$ normalized by the value of water at $4{ }^{\circ} \mathrm{C}$, which are $0.9994,1.0337$, and 0.8840 , respectively. The channel number and sampling times were chosen in the range 128-1024 and 80$400 \mu \mathrm{c}$ to obtain the suitable autocorrelation function. The integrated number was 300 . All solutions used for light scattering measurements were clarified by means of ultrafiltration through solvent resistant Durapore membrane (Millipore Filter Corporation) with a mean pore size of $0.1-0.8 \mu \mathrm{m}$, depending on the concentration of solution. The angular dependence of Rayleigh ratio of solvent was in the range $1.00-1.10$. The cell was washed with heated ethanol, using a special washing instrument constructed in our laboratory, and dried in a special box constructed to avoid dust.

\section{RESULTS AND DISCUSSION}

Figure 1 shows $H_{\mathrm{v}}$ light scattering patterns on the left whose polarization condition of the polarizer and analyzer are vertical and horizontal, respectively and the corresponding optical micrographs (cross polarizers) on the right for specimen $\mathrm{B}$ cast from acetic acid. The $H_{\mathrm{v}}$ pattern from the film, which had a thickness of $126 \mu \mathrm{m}$, shows +-type but the pattern becomes circular with increasing film thickness, reflecting multiple scattering effect. A cholesteric color could be observed for the specimen with a thickness of $714 \mu \mathrm{m}$. The color became purple in the process of evaporation of solvent and finally a pale blue color remained within the cast film. Detailed experiments showed that a cholesteric color is observed only in the case when the depth of the solution in the petri dish is such as to assure the preparation of a cast film with a thickness $>500 \mu \mathrm{m}$. As discussed before, however, such films were not suitable for light scattering experiments because of multiple scattering effects. The blue color was also observed in water under conditions which assure film thickness similar to that obtained from acetic acid but the color disappeared from the cast film after evaporation of solvent. The corresponding micrograph is indistinct because of excessful film thickness, although the focus was concentrated on the surface of the film. But the light scattering patterns indicate several rodlike textures existing in the interior of the specimen. Incidentally, color could not be observed in DMF solution but the $H_{\mathrm{v}}$ pattern of cast films has essentially the same profiles as shown in Figure 1.

For specimen $\mathrm{C}$, cholesteric color could not be observed under any condition. In order to check the time-dependence of the formation of cholesteric liquid crystals, the solutions with the concentration $(5-30) \mathrm{g} / 100 \mathrm{ml}$ had been stored for one year, but any color could not be observed in the solutions. A series of experimental results indicates that the characteristics of HPC chains in solution are sensitive to the solvent nature and molecular weight of the sample have a significant effect on the resultant cast film. That is, formation ability of liquid crystal and physical properties of the cast films are significantly affected by the characteristics of the HPC chains in solvent.

Table II shows the maximum draw ratio of films (specimens $B$ and $C$ ) cast from the indicated solution. For specimen $A$, the maximum draw ratio of films cast from DMF and acetic acid was less than 1.3 and at such a law draw ratio detailed information on the deformation mechanism was difficult to obtain. Accordingly, data for specimen $\mathrm{A}$ is not included in the table. For specimen $B$, the maximum draw ratio, $\lambda_{\max }^{\prime}$ was 1.5 for films cast from acetic acid. The cholesteric color disappeared when the sample retained to the stretcher was placed in a hot oven. The draw ratios of specimen $\mathrm{C}$ cast from water and acetic acid solutions were 7 and 4.5 times, respec- 


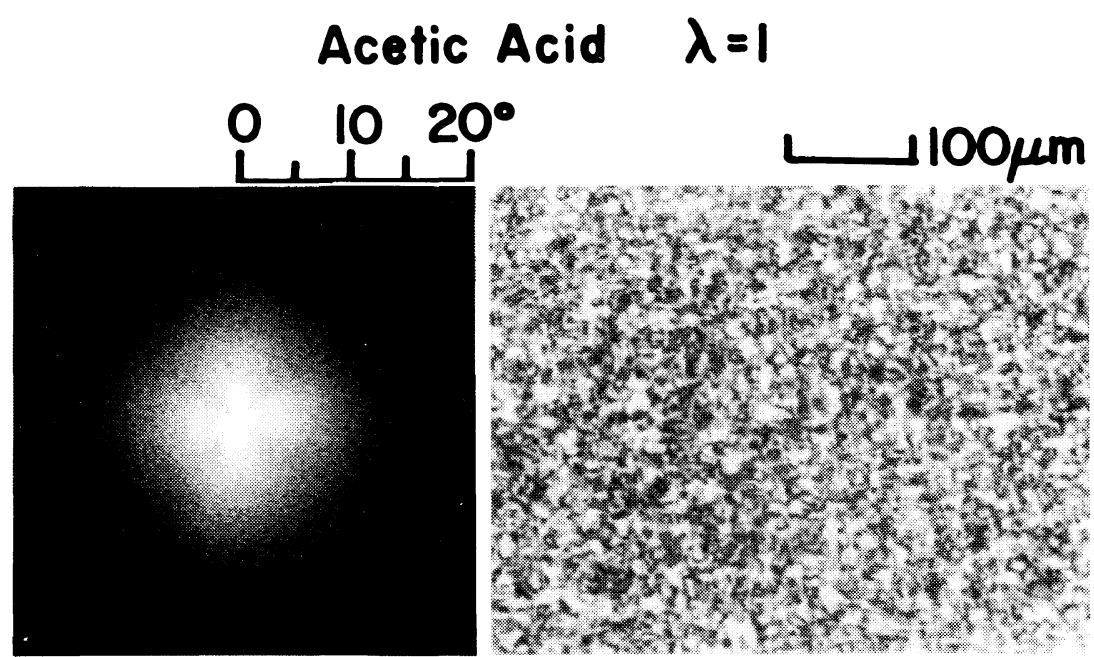

\section{(a) $126 \mu \mathrm{m}$}

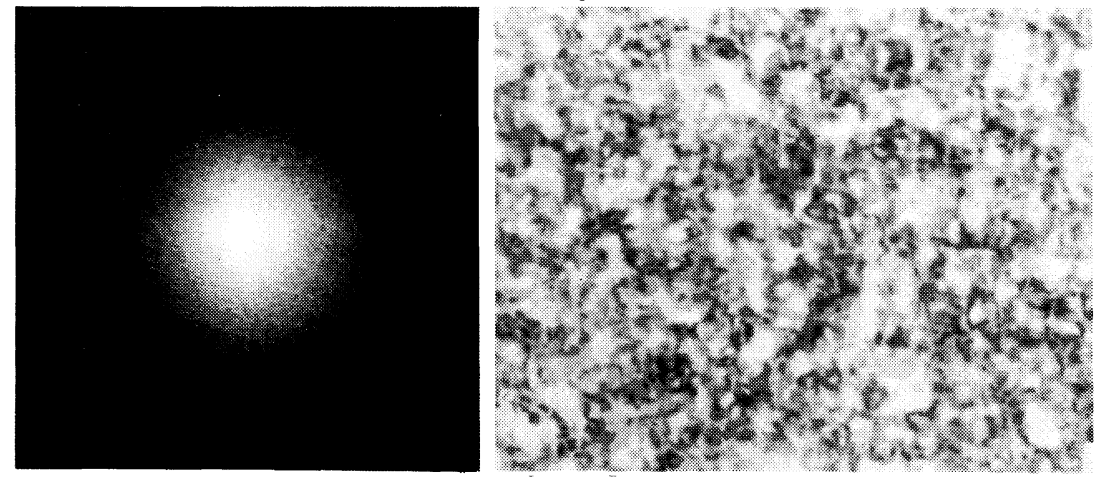

(b) $215 \mu \mathrm{m}$
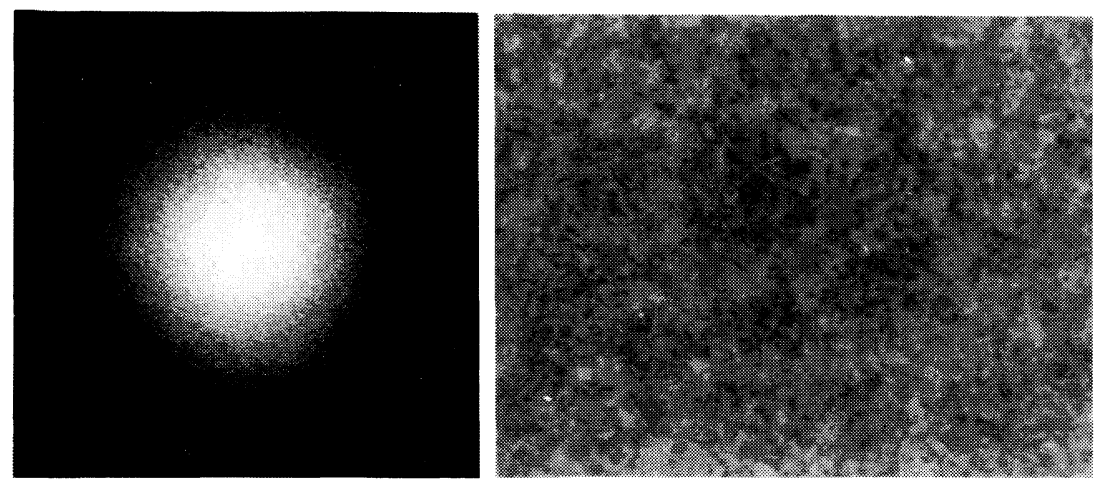

(c) $714 \mu \mathrm{m}$

Figure 1. $H_{\mathrm{v}}$ light scattering patterns and optical micrographs (crossed polarizers) of the acetic acid cast HPC films (specimen B) with different film thickness. 
Table II. Maximum draw ratio of HPC films cast from the indicated solvents

\begin{tabular}{lcc}
\hline \multicolumn{1}{c}{ Solvent } & Specimen B & Specimen C \\
\hline Water & 4.0 & 7.0 \\
$N, N$-Dimethyl- & 2.5 & 5.0 \\
$\quad$ formamide & 1.5 & 4.5 \\
Acetic acid & 1.5 \\
\hline
\end{tabular}

tively. Among the three solvents, the water cast film has the advantage in preparing films with high drawability. To elucidate this phenomenon, two treatments are employed. One is to obtain detailed information on deformation mechanism of HPC films cast under various conditions and the other is to study molecular motion and chain stiffness of HPC in solution.

Figure 2 shows $H_{\mathrm{v}}$ light scattering pattern for specimen $\mathrm{C}$ cast from acetic acid, observed as a function of draw ratio. The film thickness was about $100 \mu \mathrm{m}$. The change of the pattern with draw ratio is similar to that observed for the specimen $\mathrm{C}$ cast from water. ${ }^{2}$ That is, at initial elongation ratio $<1.8$, the meridional lobes of the pattern becomes diffuse while the equatorial lobes become sharply extended. A drastic change of the pattern arises at $\lambda=2.0$. Some $\times$-type scattering lobes appear in addition to sharp streaks in the equatorial direction, and the lobes are extended in the horizontal direction with increasing $\lambda$. Such a change is quite different from that of specimen $B$ cast from acetic acid and DMF. That is, for example, the pattern at $\lambda=1.5$ corresponding to the maximum draw ratio for specimen $B$ cast from acetic acid was quite different from that at the same draw ratio for specimen $\mathrm{C}$ (not shown in this paper). The scattering from specimen B with a thickness of $70 \mu \mathrm{m}$ exhibited very weak intensity distribution having $\times$-type lobes. The corresponding micrograph indicated rodlike textures existing in the interior of the specimen. The rods were oriented at nearly $45^{\circ}$ with respect to the stretching direction and form networks, as has been observed for poly(tetramethylene) and poly- (tetramethylene oxide) block copolymer under elongation process. ${ }^{7,8}$ The difference is probably attributed to the difference of characteristics of HPC chains for both the specimens. That is, strains of tie molecules and/or entanglements between rods for specimen B is thought to be more intense than those for specimen $C$. For specimen B, when the films cast from acetic acid were elongated in a hot oven, the maximum draw ratio was 1.5 as described before. The drawn process was non-uniform, different from the drawing behavior of specimen B cast from water solution. Instantaneous high stress occurred at initial elongation and beyond the yield point, the stress decreased drastically with draw ratio up to breaking point $(\lambda=1.5)$. Incidentally, the change of $H_{\mathrm{v}}$ patterns of specimens B cast from water, which is shown eleswhere, ${ }^{2}$ was similar to that of specimen $\mathrm{C}$ cast from acetic acid shown in Figure 2 and the maximum draw ratio reached 4 times.

Figure 3 shows the variation of birefringence with draw ratio for specimen $\mathrm{C}$ cast from the indicated solvents. The birefringence of the film cast from water is highest at each draw ratio. This is attributed to the molecular orientation but is independent of the crystallinity, since the crystallinity has an almost constant value, independent of draw ratio and the kinds of solvents as shown in Table III. X-Ray diffraction patterns (not shown in this paper) for the three kinds of cast films showed a decreasing angular spread of the strong equatorial $\left(\begin{array}{lll}1 & 0 & 0\end{array}\right)$ reflection indicating almost the same degree of the $c$-axis orientation with respect to the stretching direction. Accordingly, the different values of birefringence at each draw ratio are thought to be due to the contribution from orientation of amorphous chain segments.

Figure 4 shows the temprature dependence of the real and imaginaly parts of the complex dynamic tensile modulus of the drawn specimen. At temperature $<0^{\circ} \mathrm{C}$, the magnitude of the storage modulus $E^{\prime}$ for the water 


\section{Acetic Acid}

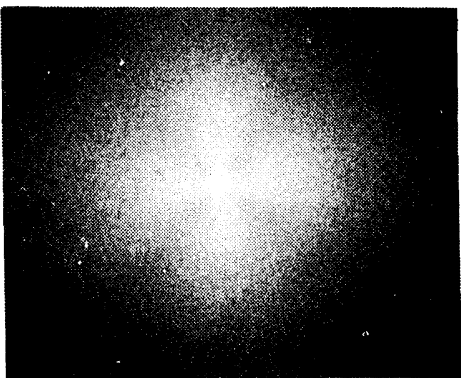

$$
\text { (a) } \lambda=1
$$

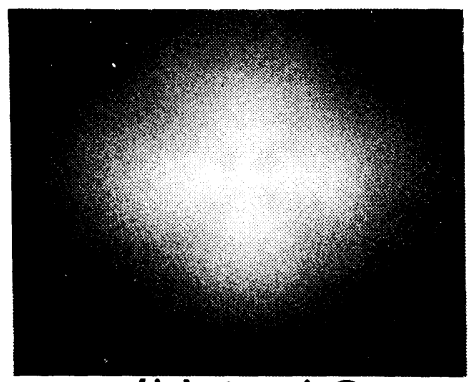

(b) $\lambda=1.2$

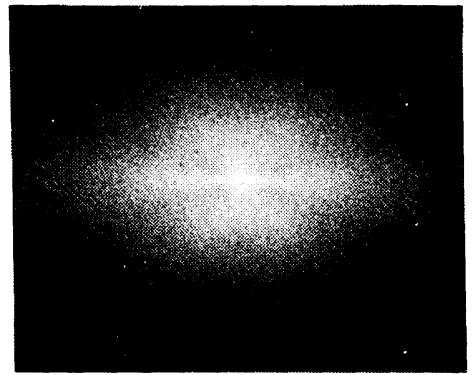

(c) $\lambda=1.5$

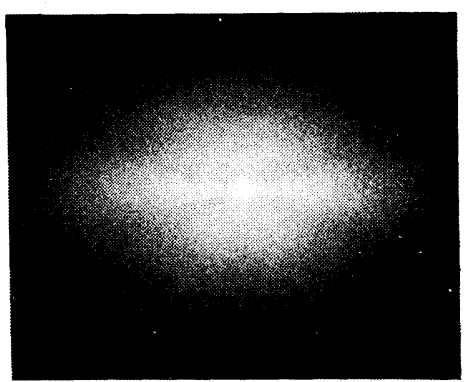

(d) $\lambda=1.8$
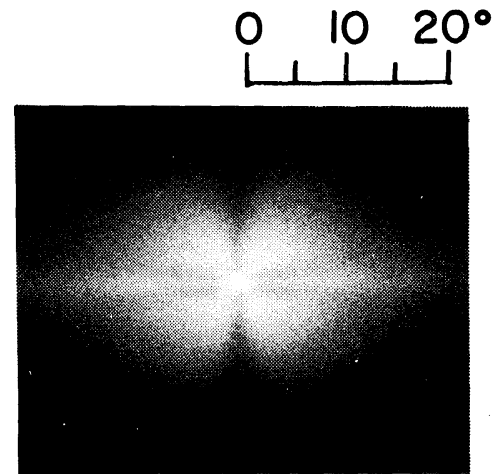

(e) $\lambda=2$

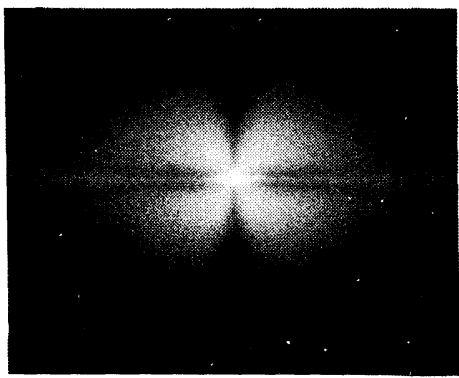

(f) $\lambda=3$

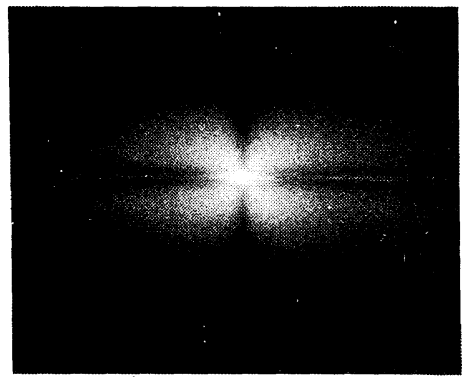

(g) $\lambda=4$

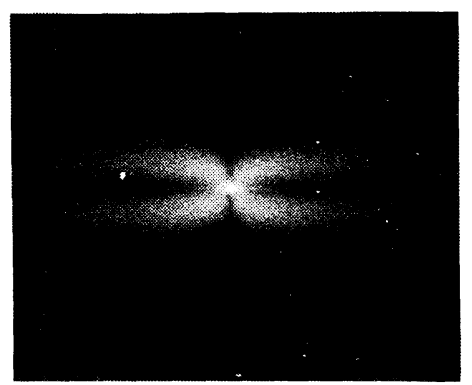

(h) $\lambda=5$

Figure 2. Change in $H_{\mathrm{v}}$ light scattering pattern from acetic acid cast film of specimen $\mathrm{C}$ at various draw ratio $\lambda$. 
Morphology and Mechanical Properties of HPC

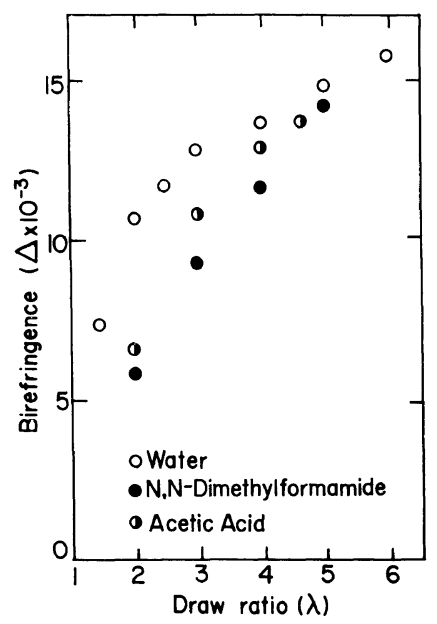

Figure 3. Birefringence of films (specimen $\mathrm{C}$ ) cast from the indicated solutions as a function of draw ratio $\lambda$.

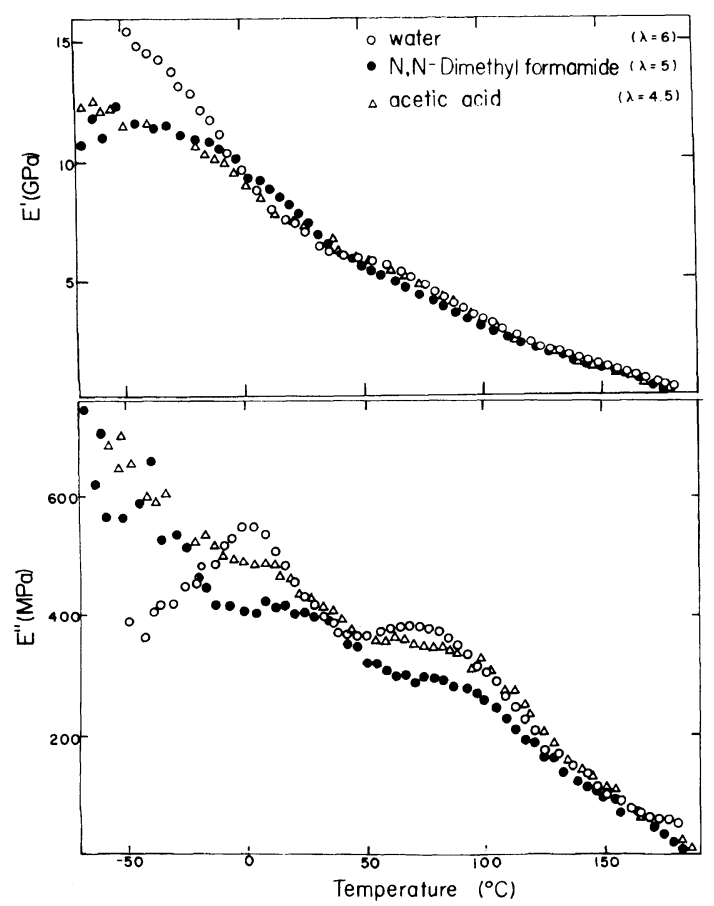

Figure 4. Temperature dependence of the storage and loss moduli of the indicated specimens.

cast film $(\lambda=6)$ increased considerably at the temperqture decreased. At temperature $>0^{\circ} \mathrm{C}$ the values of $E^{\prime}$ are almost the same regardless of the solvent and is hardly affected by the difference of the maximum draw ratio of each
Table III. Change in crystallinity of HPC films cast from the indicated solvents

\begin{tabular}{|c|c|c|c|}
\hline \multirow{2}{*}{ Solvent } & \multirow{2}{*}{ Specimen } & \multirow{2}{*}{ Draw ratio } & \multirow{2}{*}{$\frac{\text { Crystallinity }}{\%}$} \\
\hline & & & \\
\hline \multirow[t]{14}{*}{ Water } & B & 1.0 & 20.6 \\
\hline & & 1.2 & 21.2 \\
\hline & & 1.6 & 21.4 \\
\hline & & 2.4 & 21.5 \\
\hline & & 2.5 & 21.7 \\
\hline & & 3.0 & 21.8 \\
\hline & & 4.0 & 22.6 \\
\hline & $\mathrm{C}$ & 1.0 & 18.3 \\
\hline & & 2.0 & 18.9 \\
\hline & & 3.0 & 20.8 \\
\hline & & 4.0 & 21.2 \\
\hline & & 5.0 & 21.5 \\
\hline & & 6.0 & 21.7 \\
\hline & & 7.0 & 22.1 \\
\hline \multirow{7}{*}{$\begin{array}{l}N, N \text {-Dimethyl- } \\
\text { formamide }\end{array}$} & B & 1.0 & 20.3 \\
\hline & & 1.5 & 20.9 \\
\hline & $\mathrm{C}$ & 1.0 & 21.5 \\
\hline & & 2.0 & 21.5 \\
\hline & & 3.0 & 21.6 \\
\hline & & 4.0 & 22.0 \\
\hline & & 5.0 & 22.2 \\
\hline \multirow[t]{7}{*}{ Acetic acid } & B & 1.0 & 22.6 \\
\hline & & 1.5 & 23.2 \\
\hline & $\mathrm{C}$ & 1.0 & 20.4 \\
\hline & & 2.0 & 20.5 \\
\hline & & 3.0 & 20.6 \\
\hline & & 4.0 & 20.8 \\
\hline & & 4.5 & 21.2 \\
\hline
\end{tabular}

film. That is, the draw ratio does not have a significant effect on the change in the profile of $E^{\prime}$, although the birefringence values at the corresponding draw ratio are different as shown in Figure 3. This phenomenon suggests that the strains of tie molecules and/or entanglements within DMF cast film $(\lambda=5)$ and acetic acid cast film $(\lambda=4.5)$ are more intense than those within water cast film $(\lambda=6)$.

The curve of $E^{\prime \prime}$ as a function of temperature exhibits two dispersion peaks. The one is in the range $-20-50^{\circ} \mathrm{C}$ and the other in the range 
$50-100^{\circ} \mathrm{C}$. The former peak is probably associated with the transition from the glassy to the rubber-like stage due to segmental motion in the amorphous phase. The peak of the water cast film appeared distinctly on the lowest temperature side, indicating high mobility of amorphous segments, while the indistinct peak for the DMF and acetic acid cast films suggests less mobility due to the intence strains of the tie molecules and/or entanglements. Incidentally, this peak becomes indistinct for the vacuum-dried film cast from

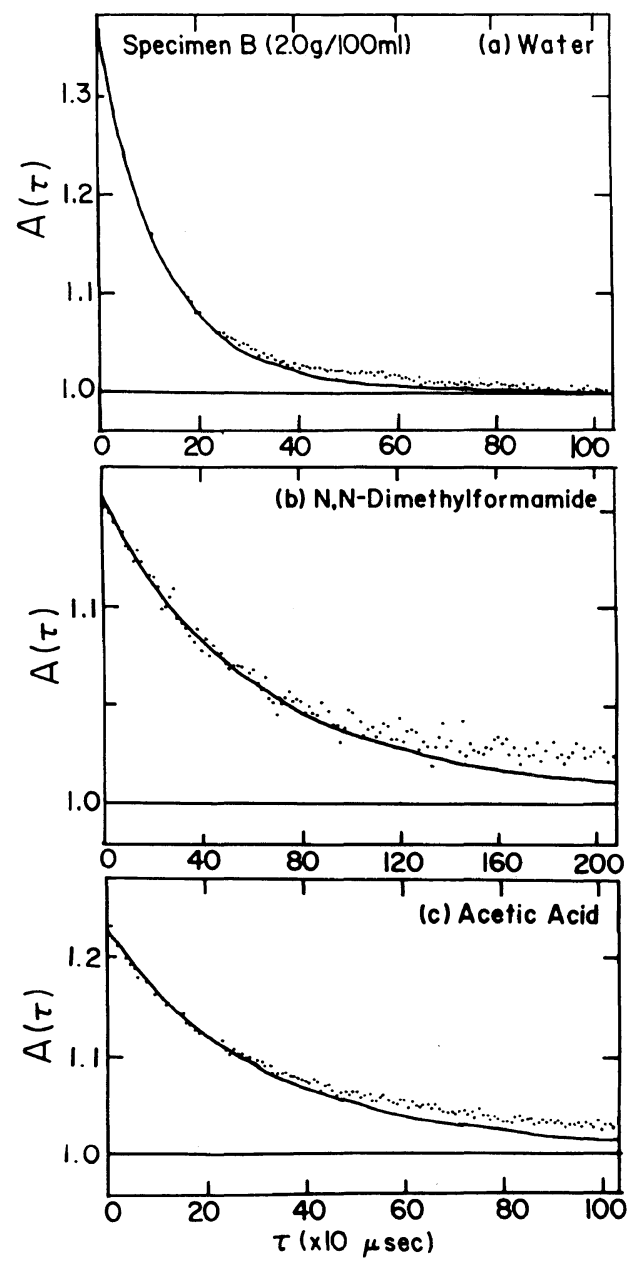

Figure 5. The autocorrelation function $A(\tau)$ plotted against delay time $\tau$ and the function (full curves) recalculated from histogram for the indicated solution of specimen $\mathbf{B}$. water. The latter peak is attributed to the high mobility of water molecules, which plays an important role as a plasticizer by disruption of inter-chain hydrogen bonds. Thus, it is reasonable that the water cast film has the most distinct peak among the three specimens. Incidentally, the influence of water to the peak profile was confirmed by such pre-experimental results that the height of the two peaks becomes more intense with increasing water content in specimens.

Figure 5 shows examples of the autocorrela-
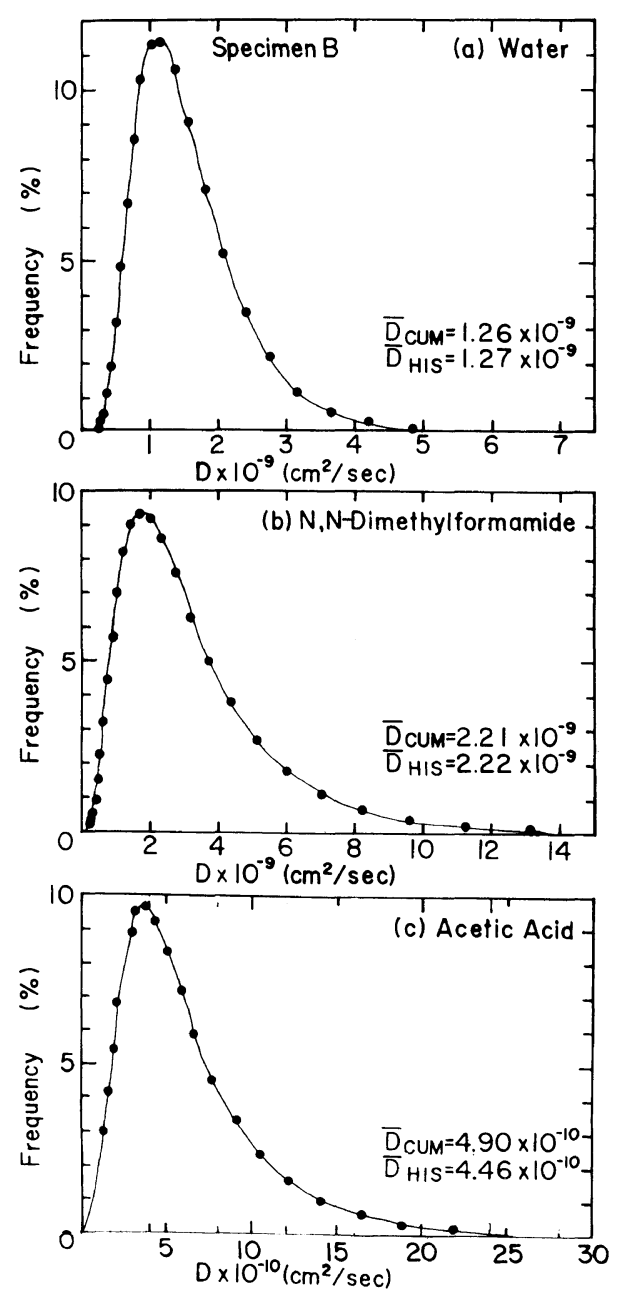

Figure 6. Distribution of the diffuse coefficient, $D$, calculated from the histgram method. $\bar{D}_{\text {CUM }}$ and $\bar{D}_{\text {HIS }}$ in the unit of $\mathrm{cm}^{2} \mathrm{~s}^{-1}$ are listed in each frame. 
Table IV. Diffuse coefficients, $\bar{D}_{\text {Cum }}$ of HPC chains in the indicated solvents calculated using the cumulation method

\begin{tabular}{|c|c|c|c|c|}
\hline \multirow{2}{*}{ Specimen } & \multirow{2}{*}{$\begin{array}{l}\text { Concentration } \\
(\mathrm{g} / 100 \mathrm{ml})\end{array}$} & \multicolumn{3}{|c|}{$\bar{D}_{\mathrm{CUM}} / \mathrm{cm}^{2} \mathrm{~s}^{-1}$} \\
\hline & & Water & $\begin{array}{l}N, N \text {-Dimethyl- } \\
\text { formamide }\end{array}$ & $\begin{array}{l}\text { Acetic } \\
\text { acid }\end{array}$ \\
\hline \multirow{5}{*}{ B } & 0.5 & $4.73 \times 10^{-9}$ & $7.54 \times 10^{-9}$ & $1.81 \times 10^{-9}$ \\
\hline & 0.8 & $3.17 \times 10^{-9}$ & $5.41 \times 10^{-9}$ & $1.36 \times 10^{-9}$ \\
\hline & 1.0 & $2.97 \times 10^{-9}$ & $4.17 \times 10^{-9}$ & $9.25 \times 10^{-10}$ \\
\hline & 1.5 & $1.92 \times 10^{-9}$ & $4.09 \times 10^{-9}$ & $6.24 \times 10^{-10}$ \\
\hline & 2.0 & $1.27 \times 10^{-9}$ & $2.28 \times 10^{-9}$ & $4.46 \times 10^{-10}$ \\
\hline \multirow{7}{*}{$\mathrm{C}$} & 0.10 & $7.76 \times 10^{-9}$ & $4.49 \times 10^{-9}$ & $1.01 \times 10^{-9}$ \\
\hline & 0.15 & $6.08 \times 10^{-9}$ & $1.94 \times 10^{-9}$ & $7.11 \times 10^{-10}$ \\
\hline & 0.20 & $3.97 \times 10^{-9}$ & $5.89 \times 10^{-10}$ & $4.84 \times 10^{-10}$ \\
\hline & 0.25 & $3.00 \times 10^{-9}$ & $4.23 \times 10^{-10}$ & $2.97 \times 10^{-10}$ \\
\hline & 0.30 & $1.37 \times 10^{-9}$ & $3.01 \times 10^{-10}$ & $2.38 \times 10^{-10}$ \\
\hline & 0.40 & $7.61 \times 10^{-10}$ & $1.92 \times 10^{-10}$ & $1.70 \times 10^{-10}$ \\
\hline & 0.50 & $4.66 \times 10^{-10}$ & $1.53 \times 10^{-10}$ & $1.20 \times 10^{-10}$ \\
\hline
\end{tabular}

tion function $A(\tau)$ at $20^{\circ} \mathrm{C}$, plotted against delay time $\tau$, for the solution of specimen $\mathbf{B}$ with a concentration of $2.0 \mathrm{~g} / 100 \mathrm{ml}$. The scattering angle was fixed to be $90^{\circ}$. The full curve is the autocorrelation function recalculated from the histograms. ${ }^{9,10}$ All the calculations were carried out by assuming an unimodal system $^{6}$ because of the broad molecular weight distribution as listed in the Table I. The recalculated curves are in good agreement with the experimental results.

Figure 6 shows examples of distribution of the diffusion coefficient, $D$, estimated from the histgram calculated from the autocorrelation function in Figure 5. In each frame, the mean value, $\bar{D}_{\text {HIS }}$, determined from the center of gravity of the distribution and the value, $\bar{D}_{\text {CuM }}$, calculated by the cumulant method are listed. The estimation by the histogram and cumulant methods has been described elsewhere in detail. ${ }^{6}$ The value of $\bar{D}_{\text {HIS }}$ are fairly in good agreement with those of $\bar{D}_{\text {CUM }}$, indicating reliability of the distribution of $D$. Then, the following values were obtained by the cumulative method.

Table IV shows the value of $\bar{D}_{\text {CuM }}$ obtained for solutions of specimens $\mathrm{B}$ and $\mathrm{C}$ as a function of concentration. The value of $\bar{D}_{\text {CUM }}$ becomes lower as the concentration increases. For specimen $\mathrm{B}$, the value at each concentration for DMF solution is the highest indicating the highest mobility of HPC chains. In contrast, the diffusion of HPC chains in acetic acid is lowest. Here it should be noted that the above relationship between $\bar{D}_{\text {CUM }}$ in water and DMF solutions is reversed for specimen $\mathrm{C}$. This phenomenon is probably related to draining or non-draining effect of solvents in HPC solutions associated with molecular weight distribution and the degree of substitution. Actually, the introduction of draining effect has been successful in estimating the unperturbed chain dimension of cellulose and its derivative solutions. ${ }^{11}$

The results in Table IV suggest that the ability of HPC to form liquid crystals depends on the properties of HPC chains in solution. The HPC chains in DMF could not form liquid crystal even for specimen $\mathrm{B}$ as indicated before. Based on these results, it may be expected that active diffusion of HPC chains in DMF hampers formation of liquid crystal, while the HPC chains in acetic acid and water can form liquid crystal easily because molecular diffu- 
sion of HPC chains in these solvents is less pronounced than that in DMF. Interestingly, HPC chains in acetic acid with the lowest molecular mobility retains cholesteric color within the dried film after evaporation of solvent, as described before. This supports the hypothesis that the molecular mobility of HPC chains in solution plays an importnat role in the formation of liquid crystals.

A question, however, arises as to why all the solutions of specimen C can't form liquid crystals in spite of the fact that the values of the diffusion coefficient are lower than those for acetic acid solutions of specimen $B$ at the same concentration, $0.5 \mathrm{~g} / 100 \mathrm{ml}$. Apart from the molecular mobility discussed in term of the same molecular weight level, it may be implied that relaxation time to attain an equilibrium state becomes longer as chain length increases and then this phenomenon hampers the formation of liquid crystals in a short period. Furthermore it has been reported that the persistent length, which is clearly related to the form ability of liquid crystals, is indepdnent of molecular weight.

To facile understanding the more detailed characteristic dependence of HPC chains on solvent nature, the ratio, $\left(\left\langle R_{0}^{2}\right\rangle /\left\langle R_{\mathrm{f}}^{2}\right\rangle\right)^{1 / 2}$, was obtained by using method of Wirick and Waldman, ${ }^{5}$ in which $\left\langle R_{0}^{2}\right\rangle^{1 / 2}$ is the unperturbed root mean square end-to-end distance of HPC chains in solution associated with measurements by elastic light scattering, and $\left\langle R_{\mathrm{f}}^{2}\right\rangle^{1 / 2}$ is the unperturbed root mean square end-to-end distance for 1,4'-polysaccharide molecules. This concept is based on the assumption that the ratio $\left(\left\langle R_{0}^{2}\right\rangle /\left\langle R_{\mathrm{f}}^{2}\right\rangle\right)^{1 / 2}$ is hardly affected by the small difference of MS values between specimen B and C. Figure 7 shows an example of the Zimm plot for specimen $\mathrm{B}$ in acetic acid at $20^{\circ} \mathrm{C}$. The distorted profile is probably due to the broad molecular weight distribution. For DMF, the Zimm plot could not be made because of extensive scattering of the experimental data points. The Zimm plots for the other solvents showed

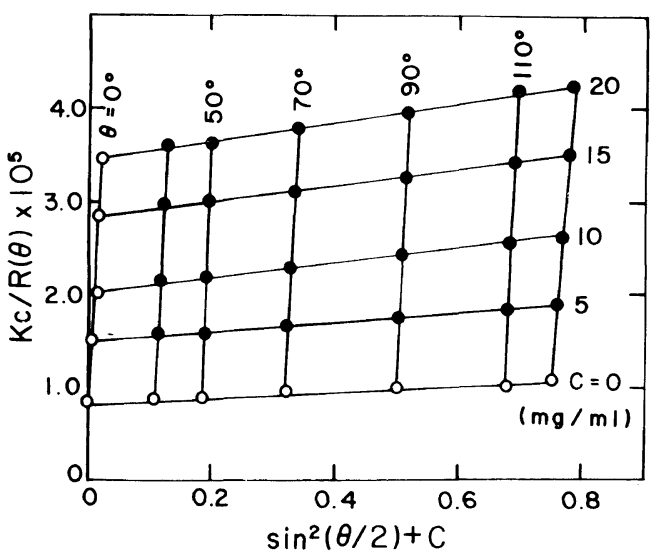

Figure 7. Zimm plot of specimen $B$ in acetic acid solution for specimen $B$.

Table V. The ratio $\left\langle R_{0}^{2}\right\rangle^{1 / 2} /\left\langle R_{\mathrm{f}}^{2}\right\rangle^{1 / 2}$ for specimens $\mathrm{B}$ and $\mathrm{C}$ in water and acetic acid

\begin{tabular}{lcc}
\hline & Specimen B & Specimen C \\
\hline Water & 4.6 & - \\
Acetic acid & 5.9 & 4.1
\end{tabular}

distorted profiles similar to that in Figure 7. To check the reproducibility of the data, light scattering measurements were carried out repeatedly. For specimen B, the value of $\bar{M}_{w}$, $19.2 \times 10^{4}$, obtained from acetic acid was close to that obtained from water. Unfortunately, several measurements for specimen $\mathrm{C}$ showed that the values of $\bar{M}_{w}$ obtained from both solutions are scattered and this tendency is considerable for water. The value of $M_{w}$ measured for acetic acid was in the range $105-125 \times 10^{4}$. Among several values, the employed value was $\bar{M}_{w}=117 \times 10^{4}$, being close to the values $119.5 \times 10^{4}$ obtained by gel permeation listed in Table I and consequently the second virial coefficient of $A_{2}$ and $Z$-mean radius of gyration $\left\langle S_{2}\right\rangle_{z}^{1 / 2}$ measured simulataneously were adopted as the corresponding values. On the basis of the same method proposed by Wirick and Waldman ${ }^{5}$ as described in the Appendex, the values of $\left(\left\langle R_{0}^{2}\right\rangle /\left\langle R_{\mathrm{f}}^{2}\right\rangle\right)^{1 / 2}$ are calculated in Table V. The 
value of $\left(\left\langle R_{0}^{2}\right\rangle /\left\langle R_{\mathrm{f}}^{2}\right\rangle\right)^{1 / 2}$ for specimen $\mathrm{C}$ is lower than those for specimen $B$. This means that the rigidity of HPC chain becomes less pronounced as the molecular weight increases, indicating a transition from a hindered random coil at high molecular weight to a relatively stiff rod at low molecular weight as pointed by Wirick et al. ${ }^{5}$ For specimen $\mathrm{B}$, the value of $\left(\left\langle R_{0}^{2}\right\rangle /\left\langle R_{\mathrm{f}}^{2}\right\rangle\right)^{1 / 2}$ in acetic acid is higher than that in water. This is in good agreement with the results as listed in Table IV. indicating a lower mobility of HPC chains in acetic acid than in water. All the values of $\left(\left\langle R_{0}^{2}\right\rangle /\left\langle R_{\mathrm{f}}^{2}\right\rangle\right)^{1 / 2}$ are higher than 4 . Especially, in acetic acid solution, the value of specimen B is close to 6 which is much higher than the value proposed by Wirick and Waldman. ${ }^{5}$ According to their report, the value was 4.4 in ethanol for a specimen of $\overline{D P}=420$. Thus, the highest value of molecular expansion factor of HPC chains in acetic acid is thought to play an important role to form a stable cholesteric liquid crystals discussed before.

Judging from Table V, drawability becomes less pronounced on increasing the ratio, $\left\langle R_{0}^{2}\right\rangle^{1 / 2} /\left\langle R_{\mathrm{f}}^{2}\right\rangle^{1 / 2}$. Namely, the draw ratio of specimen $B$ cast from water and acetic acid was 4 and 1.5 respectively, as listed in Table II and the corresponding ratio $\left\langle R_{0}^{2}\right\rangle^{1 / 2} /\left\langle R_{\mathrm{f}}^{2}\right\rangle^{1 / 2}$ for water is lower than that for acetic acid as listed in Table $\mathrm{V}$. The draw ratio of specimen $\mathrm{C}$ cast from acetic acid is close to that of specimen $B$ cast from water and the corresponding ratios $\left\langle R_{0}^{2}\right\rangle^{1 / 2} /\left\langle R_{\mathrm{f}}^{2}\right\rangle^{1 / 2}$ of both specimens show little difference. In addition to draw ratio, the ratio $\left\langle R_{0}^{2}\right\rangle^{1 / 2} /\left\langle R_{\mathrm{f}}^{2}\right\rangle^{1 / 2}$ is also effective to the deformation mechanism of rodlike texture within the cast film. As discussed in relation to Figure 2, the light scattering pattern of specimen $C$ cast from acetic acid was similar to that of spcimen B cast from water. In spite of such significant results, the crystallity of the cast films are hardly affected by the ratio $\left\langle R_{0}^{2}\right\rangle^{1 / 2} /\left\langle R_{\mathrm{f}}^{2}\right\rangle^{1 / 2}$ as listed in Table III.

A series of experimental results demonstrated that one of the important factors for determining the morphology and deformation mechanism of cast films has to be traced to the characteristics of molecular chains in solution. Unfortunately, this concept contains somewhat ambiguous points because of the broad molecular weight distribution of the test specimens and the complexity of the monomer unit of HPC associated with the molar substitution. Nevertheless, trends shown by the experimental data are not unreasonable, and encourages further attempts to relate the drawability of solution cast films to the solution properties of the chain molecules.

\section{CONCLUSION}

The morphology and drawability of HPC cast film is dpendent upon solvent nature and molecular weight of sample. The maximum draw ratios, for example, of cast film from acetic acid were 1.5 and 4.0 for specimens B $\left(\bar{M}_{w}=19.2 \times 10^{4}\right)$ and specimen C $\left(\bar{M}_{w}=\right.$ $\left.119.5 \times 10^{4}\right)$, respectively, while those of water cast film related 4.0 and 7.0. To study this phenomenon, elastic and inelastic light scattering measurements were carried out. These expermental results showed that in comparison with the same specimen, the drawability of cast film become more pronounced as diffusion coefficient and molecular expansion factor of HPC chains in solvent increase, indicating that the morphology and deformation mechanism of cast film has to be traced to the characteristics of the HPC chains in solution.

Acknowledgements. We thank Prof. Y. Tsunashima, Institute for Chemical Research Kyoto University, Uji, Japan for valuable comments and suggestions for measuring autocorrelation functions. We are also indebted to Prof. S. Suto, Faculty of Engineering, Yamagata University for measuring molar substitution (MS), $\bar{M}_{w}$ and $\bar{M}_{n}$. We are also grateful to Prof. Manley of McGill University, Canada, for kindly reading the manuscript and making numerous suggestions. 


\section{APPENDIX}

The estimation of $\left(\left\langle R_{0}^{2}\right\rangle /\left\langle R_{\mathrm{f}}^{2}\right\rangle\right)^{1 / 2}$ was done by the same method employed for HPC solutions by Wrick and Waldman. ${ }^{5}$ The unperturbed root mean square end to end distance $\left\langle R_{0}^{2}\right\rangle_{z}^{1 / 2}$ was calculated for the solution using the relation

$$
\left\langle R_{0}^{2}\right\rangle_{z}^{1 / 2}=\left\langle R^{2}\right\rangle / \alpha
$$

where $\left\langle R_{2}\right\rangle_{z}^{1 / 2}$ is the root square end to end distance which can be estimated from the $z$-average mean square radius of gyration $\left\langle S^{2}\right\rangle_{z}^{1 / 2}$ measured by elastic light scatteing through an approximate relation: $\left\langle S^{2}\right\rangle_{z}=$ $\left\langle R^{2}\right\rangle_{z} / 6 . \alpha$ is the molecular expansion factor and is esimated according to Orofino and Flory, ${ }^{12}$ from the following equations:

$$
A_{2}=\left(\frac{16 \pi}{3^{3 / 2}}\right)\left(\frac{N\left\langle S^{2}\right\rangle_{z}^{3 / 2}}{\bar{M}_{z}^{2}}\right) \ln \left[1+\frac{\sqrt{\pi}}{2}\left(\alpha^{2}-1\right)\right]
$$

where $A_{2}$ is the second virial coefficient and $N$ is the Avogadro number. $\bar{M}_{z}, z$-average molecular weight was estimated from $\bar{M}_{w}$ and $M_{n}$ assuming that the samples have ShulzZimm type molecular weight distribution. ${ }^{14}$

The values of $A_{2}$ and $\left\langle S^{2}\right\rangle_{z}$ were measured by elastic light scattering measurements. For specimen $\mathrm{B}$, the values of $A_{2}$ and $\left\langle S^{2}\right\rangle_{z}^{1 / 2}$ are estimated to be $6.56 \times 10^{-4} \mathrm{~cm}^{3} \cdot \mathrm{mol} \cdot \mathrm{g}^{-2}$ and $384 \AA$, respectively, in acetic acid, while the corresponding values in water were estimated to be $7.15 \times 10^{-5} \mathrm{~cm}^{3} \cdot \mathrm{mol} \cdot \mathrm{g}^{-2}$ and $284 \AA$ respectively. The values of $M_{w}$ of specimen C in water were scattered. For acetic acid, the values of $A_{2}$ and $\left\langle S^{2}\right\rangle_{z}^{1 / 2}$ were estimated to be $1.2 \times 10^{-4} \mathrm{~cm}^{3} \cdot \mathrm{mol} \cdot \mathrm{g}^{-2}$ and $650 \AA$, respectively.

The unperturbed root mean square end to end distance, $\left\langle R_{\mathrm{f}}^{2}\right\rangle^{1 / 2}$, for 1,4-polysaccharide molecules, assuring free rotation of the chain units, may be calculated from the relation according to Eliezer and Hayman. ${ }^{13}$ That is

$$
\left\langle R_{\mathrm{f}}^{2}\right\rangle^{1 / 2}=7.75 \overline{D P}_{z}^{1 / 2}
$$

where $D P_{z}$ is $z$-average degree of polymerization and is related to weight average degree of polymerization $\overline{D P}_{w}$ by multiplying $\bar{M}_{z} / \bar{M}_{w}$. $\overline{D P}_{w}$ is related to

$$
[\eta]=7.2 \times 10^{-3} \overline{D P}_{w}^{0.900}
$$

wher, $[\eta]$ (unit: $100 \mathrm{mlg}^{-1}$ ) is the limiting viscosity number of HPC specimen in ethanol at $25^{\circ} \mathrm{C}$. This value was obtained from the relationship between the reduced viscosity and concentration in ethanol solution for specimens $B$ and $C$.

\section{REFERENCES}

1. R. J. Samuels, J. Polym. Sci., A-2, 1, 1197 (1969).

2. M. Matsuo and N. Yanagida, Polymer, 32, 2561 (1991).

3. M. B. Rhodes and R. S. Stein, J. Polym. Sci., A-2, 1, 1539 (1969)

4. P. Smith, P. J. Lemstra, and H. C. Booij, J. Polym. Sci., Polym. Phys. Ed., 19, 877 (1981).

5. H. G. Wirick and M. H. Waldman, J. Appl. Polym. Sci., 14, 579 (1970).

6. N. Yanagida and M. Matsuo, Polymer, 33, 996 (1992).

7. C. Sawatari, M. Iida, and M. Matsuo, Macromolecules, 17, 1765 (1984).

8. M. Iida, C. Sawatari, and M. Matsuo, J. Chem. Soc., Faraday Trans. 2, 80, 1599 (1984).

9. B. Chu, "Laser Light Scattering," Academic Press, New York, 1974.

10. E. Gulari, Y. Tsunashima, and B. Chu, Polymer, 20, 347 (1979).

11. For example, K. Kamide and M. Saito, Adv. Polym. Sci., 83, 1 (1987).

12. T. A. Orofino and P. Florly, J. Chem. Phys., 39, 1067 (1955).

13. I. Eliezer and H. G. Hayman, J. Polym. Sci., 23, 387 (1957).

14. B. H. Zimm, J. Chem. Phys, 16, 1099 (1948). 